\title{
Inverse Shadowing and Weak Inverse Shadowing Property
}

\author{
B. Honary, Alireza Zamani Bahabadi \\ Department of Mathematics, Ferdowsi University of Mashhad, Mashhad, Iran \\ Email: honary@math.um.ac.ir, zamany@um.ac.ir
}

Received February 25, 2012; revised April 5, 2012; accepted April 12, 2012

\begin{abstract}
In this paper we show that an $\Omega$-stable diffeomorphism $f$ has the weak inverse shadowing property with respect to classes of continuous method $\theta_{s}$ and $\theta_{c}$ and some of the $\Omega$-stable diffeomorphisms have weak inverse shadowing property with respect to classes $\mathcal{T}_{0}$. In addition we study relation between minimality and weak inverse shadowing property with respect to class $\mathcal{T}_{0}$ and relation between expansivity and inverse shadowing property with respect to class $\mathcal{T}_{0}$.
\end{abstract}

Keywords: Inverse Shadowing Property; Minimal Homeomorphism; $\delta$-Method; Positive Expansive

\section{Introduction}

Inverse shadowing was introduced by Corless and Pilyugin [1] and also as a part of the concept of bishadowing by Diamond et al. [2]. Kloeden, Ombach and Pokroskii [3] defined this property using the concept of $\delta$-method. One can also see [4-7] for more information about the concept of $\delta$-method. Authors in [8] studied on locally genericity of weak inverse shadowing with respect to class $\mathcal{T}_{0}$. For flows, there are lots of existing work on finding the minimal sets in a systems with shadowing property. See for example [9-12]. In this paper we study diffeomorphisms with weak inverse shadowing property with respect to class as $\theta_{s}, \theta_{c}$ and $\mathcal{T}_{0}$. First we show that an $\Omega$-stable diffeomorphism $f$ has weak inverse shadowing property with respect to classes of continuous method $\theta_{s}$ and $\theta_{c}$ (Theorem 1) and some $\Omega$-stable diffeomorphisms have weak inverse shadowing property with respect to classes $\mathcal{T}_{0}$ (Theorem 2). In addition we study relation between minimality and weak inverse shadowing property with respect to class $\mathcal{T}_{0}$ and show that a chain transitive homeomorphism $f$ on compact metric space $X$ is minimal if and only if it has weak inverse shadowing property with respect to class $\mathcal{T}_{0}$ (Theorem 3 ). Finally we study relation between positively expansive and inverse shadowing property with respect to class $\mathcal{T}_{0}$ and show that if $f$ has inverse shadowing property with respect to class $\mathcal{T}_{0}$, then $f$ is not positive expansive (Theorem 4).
Let $(X, d)$ be a compact metric space and let $f: X \rightarrow X$ be a homeomorphism (a discrete dynamical system on $X$ ). A sequence $\left\{x_{n}\right\}_{n \in \mathbb{Z}}$ is called an orbit of $f$, denote by $o(x, f)$, if for each $n \in \mathbb{Z}$, $x_{n+1}=f\left(x_{n}\right)$ and is called a $\delta$-pseudo-orbit of $f$ if

$$
d\left(f\left(x_{n}\right), x_{n+1}\right) \leq \delta, \forall n \in \mathbb{Z} .
$$

Denote the set of all homeomorphisms of $X$ by $Z(X)$. In $Z(X)$ consider the complete metric

$$
\begin{aligned}
& d_{0}(f, g)=\max \left\{\max _{x \in X} d(f(x), g(x)),\right. \\
& \left.\max _{x \in X} d\left(f^{-1}(x), g^{-1}(x)\right)\right\},
\end{aligned}
$$

which generates the $C^{0}$-topology.

Let $X^{\mathbb{Z}}$ be the space of all two sided sequence $\xi=\left\{x_{n}: n \in \mathbb{Z}\right\}$ with elements $x_{n} \in X$, endowed with the product topology. For $\delta>0$ let $\Phi_{f}(\delta)$ denote the set of all $\delta$-pseudo orbits of $f$.

A mapping $\varphi: X \rightarrow \Phi_{f}(\delta) \subset X^{\mathbb{Z}}$ is said to be a $\delta$-method for $f$ if $\varphi(x)_{0}=x$, where $\varphi(x)_{0}$ is the 0 -component of $\varphi(x)$. If $\varphi$ is a $\delta$-method which is continuous then it is called a continuous $\delta$-method. The set of all $\delta$-methods (resp. continuous $\delta$-methods) for $f$ will be denoted by $\mathcal{T}_{0}(f, \delta)$ (resp. $\left.\mathcal{T}_{c}(f, \delta)\right)$. If $g: X \rightarrow X$ is a homeomorphism with $d_{0}(f, g)<\delta$, then $g$ induces a continuous $\delta$-method $\varphi_{g}$ for $f$ defined by

$$
\varphi_{g}(x)=\left\{g^{n}(x): n \in \mathbb{Z}\right\} .
$$

Let $\mathcal{T}_{h}(f, \delta)$ denote the set of all continuous $\delta$ - 
methods $\varphi_{g}$ for $f$ which are induced by $g \in Z(M)$ with $d_{0}(f, g)<\delta$.

Let $A \subseteq M$ and $A \neq \phi$, a homeomorphism $f$ is said to have the inverse shadowing property with respect to the class $\mathcal{T}_{\alpha}, \alpha=0, c, h$, in $A$ if for any $\varepsilon>0$ there is $\delta>0$ such that for any $\delta$-method $\varphi$ in $\mathcal{T}_{\alpha}(f, \delta)$ and any point $x \in A$ there exists a point $y \in M$ for which

$$
d\left(f^{n}(x), \varphi(y)_{n}\right)<\varepsilon, n \in \mathbb{Z} .
$$

A homeomorphiosm $f$ is said to have weak inverse shadowing property with respect to the class $\mathcal{T}_{\alpha}, \alpha=0$, $c, h$, in $A$ if for any $\varepsilon>0$ there is $\delta>0$ such that for any $\delta$-method $\varphi$ in $\mathcal{T}_{\alpha}(f, \delta)$ and any point $x \in A$ there exists a point $y \in M$ for which

$$
\varphi(y) \subset N_{\varepsilon}(o(x, f)) .
$$

Fix $\delta>0$. A continuous $\delta$-method of class $\theta_{s}$ for the diffeomorphism $f$ is a sequence $\Psi=\left\{\psi_{k}: k \in Z\right\}$, where any $\psi_{k}$ is a continuous mapping $\psi_{k}: M \rightarrow M$ such that

$$
\max _{x \in M} d\left(\psi_{k}(x), f(x)\right)<d, k \in Z .
$$

A sequence $\xi=\left\{x_{k} \in M: k \in Z\right\}$ is a pseudo-orbit generated by a continuous $d$-method $\Psi=\left\{\psi_{k}\right\}$ of a class $\theta_{s}$ if

$$
x_{k+1}=\psi_{k}\left(x_{k}\right), k \in Z \text {. }
$$

Fix $\delta>0$. A continuous $\delta$-method of class $\theta_{c}$ for the diffeomophism $f$ is a sequence $\Psi=\left\{\psi_{k}: k \in Z\right\}$, with $\psi_{0}(x)=x$ for $x \in M$ and such that any $\psi_{k}$ is a continuous mapping $\psi_{k}: M \rightarrow M$ with the property

$$
\max _{x \in M} d\left(f\left(\psi_{k}(x)\right), \psi_{k+1}(x)\right)<\delta, k \in Z .
$$

A sequence $\xi=\left\{x_{k} \in M: k \in Z\right\}$ is a pseudo-orbit generated by a continuous $\delta$-method $\Psi=\left\{\psi_{k}\right\}$ of class $\theta_{c}$ if

$$
x_{k}=\psi_{k}\left(x_{0}\right), k \in Z .
$$

If a sequence is generated by $\theta_{c}$ or $\theta_{s}$ we briefly write $\xi \in G \Psi$.

A diffeomorphism $f$ is said to have (weak) inverse shadowing property if for any $x \in M$ and $\varepsilon>0$ there exists $\delta>0$ such that, for any continuous $\delta$-method $\Psi$, we can find a pseudo-orbit $\xi \in G \Psi$ satisfying the inequalities

$$
\begin{gathered}
d\left(f^{k}(x), x_{k}\right)<\varepsilon, k \in Z . \\
\left(\left\{x_{k}\right\} \subset N_{\varepsilon}(O(x, f)) .\right)
\end{gathered}
$$

Pilyugin [5] showed that a structurally stable diffeomoriphism has the inverse shadowing property with respect to classes of continuous method, $\theta_{c}$ and $\theta_{s}$. He also showed that any diffeomorphism belonging to the $C^{1}$-interior of the set of diffeomorphisms having the inverse shadowing property with respect to classes of continuous method, $\theta_{c}$ and $\theta_{s}$ is structurally stable.

\section{Diffeomorphisms with Weak Inverse Shadowing Property with Respect to Class $\theta_{s}, \theta_{c}$ and $\mathcal{T}_{0}$}

In this section we show that an $\Omega$-stable diffeomorphism $f$ has the weak inverse shadowing property with respect to classes of continuous methods $\theta_{s}$ and $\theta_{c}$ and if we impose some condition on an $\Omega$-stable diffeomorphism, then it has weak inverse shadowing property with respect to classes $\mathcal{T}_{0}$.

Theorem 1 If a diffeomorphism $f$ is $\Omega$-stable, then it has the weak inverse shadowing property with respect to both classes $\theta_{c}$ and $\theta_{s}$.

Before proving this main result, let us briefly recall some definitions. A diffeomorphism $f: M \rightarrow M$ is called $\Omega$-stable if there is a $C^{1}$-neighborhood $U$ of $f$ such that for any $g \in U,\left.g\right|_{\Omega(g)}$ is topologically conjugate to $\left.f\right|_{\Omega(f)}$.

A diffeomorfphism $f$ is called an Axiom A system if $\Omega(f)$ is hyperbolic and if $\Omega(f)=\overline{\text { pref }}$. Axiom $A$ and no-cycle systems are $\Omega$-stable [13].

Let $f$ be an Axiom $A$ diffeomorphism of $M$. By the Smale spectral Decomposition Theorem, the nonwandering set $\Omega(f)$ an e represented as a finite union of basic sets $\Lambda_{i}$.

$$
\Omega(f)=\Lambda_{1} \cup \cdots \cup \Lambda_{k}
$$

In the proof of theorem 1 in [5], Pilyugin has used the following statement.

If a $C^{1}$-diffeomorphism $f$ satisfies Axiom $A$ and the strong transversality condition, then there exist constants $C>0$ and $\lambda \in(0,1)$ and linear subspace $S(p)$, $U(p)$ of $T_{p} M$ for $p \in M$ such that

$$
\begin{aligned}
& T_{p} M=S(p) \oplus U(p), \\
& D f(p) S(p) \subset S(f(p)), \\
& D f^{-1}(p) U(p) \subset U\left(f^{-1}(p)\right),
\end{aligned}
$$

and

$$
\begin{aligned}
& \left|D f^{k}(p) V\right| \leq C \lambda^{k}|V| \text { for } k \geq 0 \text { and } V \in S(p), \\
& \left|D f^{k}(p) V\right| \leq C \lambda^{k}|V| \text { for } k \geq 0 \text { and } V \in U(p),
\end{aligned}
$$

if $P(p)$ and $Q(p)$ are the projectors in $T_{p} M$ onto $S(p)$ parallel to $U(p)$ and onto $U(p)$ parallel to $S(p)$, respectively, then 


$$
\|P(p)\| \leq C \text { and }\|Q(p)\| \leq C
$$

(here $\|$.$\| is the operator norm).$

Conditions (1), (2) and (3) play a basic role in the proof of theorem 1 in [5]. If $\Lambda_{i}$ is a basic set then we can see for every $x \in \Lambda_{i}$, conditions (1), (2) hold. Since $\|f(x)\|$ is bounded for $x \in \Lambda_{i}$, standard reasening shows (see, for example, Lemma 12.1 in [14]) that there exists a constant $C$ for which inequalities (3) hold. Hence similar to the proof of theorem 1 in [5], $f$ has the inverse shadowing property with respect to classes $\theta_{\mathrm{s}}$ and $\theta_{c}$ on $\Lambda_{i}$. The following two propositions are well known (proposition 1 is the classical Birkhoff theorem [13], for proofs of statements similar to proposition 2, see [15], for example).

proposition 1 Let $f$ be a homeomorphism of a compact topological space $X$ and $U$ be a neighborhood of its nonwandering set. Then there exists a positive integer $N$ such that

$$
\operatorname{Card}\left(k: f^{k}(x) \notin U\right) \leq N
$$

for every $x \in X$, where Card $A$ is the cardinality of a set $A$.

In the following proposition, we assume that $f$ is an $\Omega$-stable diffeomorphism of a closed smooth manifold.

proposition 2 If $\Lambda_{i}$ is a basic set, then for any neighborhood $U$ of $\Lambda_{i}$ there exists neighborhood $V$ with the following property: if for some $x \in V$ and $m>0, \quad f^{m}(x) \notin U$, then $f^{m+k}(x) \notin V$ for $k \geq 0$.

Lemma 1 Let $f$ be an $\Omega$-stable diffeomorphism and $\Omega(f)=\Lambda_{1} \cup \cdots \cup \Lambda_{k}$ be the Smale Spectral Decomposition. Let $U_{i}$ be a neighborhood of $\Lambda_{i}$ for $i=1, \cdots, k$. Then for any $x \in M$ there exists $N_{0} \in N$ and $U_{i}$ for some $1 \leq i \leq k$, such that

$$
\left\{f^{N_{0}+k}(x)\right\}_{k \geq 0} \subset U_{i},
$$

and similarly there exists $U_{j}$

$$
\left\{f^{-N_{0}+k}(x)\right\}_{k \geq 0} \subset U_{j},
$$

Proof. Suppose that the lemma is not true for some $x \in M$. Let $V_{i}$ be a neighborhood of $\Lambda_{i}$ as in proposition 2. Proposition 1 shows that there exists $n_{i} \in N$ such that $f^{n_{i}}(x) \in V_{i}$ for some $1 \leq i \leq k$. By assumption there exists $m_{i} \in N, m_{i}>n_{i}$ such that $f^{m_{i}}(x) \notin U_{i}$. By proposition 2, $f^{m_{i}+n}(x) \notin V_{i}$ for $n \geq 0$. Thus using proposition 1, there exists $n_{j} \in N, n_{j}>m_{i}$ such that $f^{n_{j}}(x) \in V_{j}$ for some $1 \leq j \leq k \quad j \neq i$ and there exists $m_{j} \in N, m_{j}>n_{j}$ such that $f^{m_{j}}(x) \notin U_{j}$. By proposition $2, f^{m_{j}+n}(x) \notin V_{j}$ for $n \geq 0$. This process show that

$$
\operatorname{Card}\left\{n: f^{n}(x) \notin U:=\bigcup_{i=1}^{k} V_{i}\right\}=\infty
$$

contradicting proposition 1. Proof of

$$
\left\{f^{-N_{0}+k}(x)\right\}_{k \geq 0} \subset U_{j},
$$

is similar.

Proof of theorem 1. Let $x \in M$ and $\varepsilon>0$ be arbitrary. Let $U_{i}$ be a neighborhood of $\Lambda_{i}$ for $i=1, \cdots, k$, such that shadowing property hold for them. By lemma 1 there exists a positive number $N_{0}$, such that $\left\{f^{n}(x)\right\}_{n \geq N_{0}} \subset U_{i}$ for some $1 \leq i \leq k$. Since $M$ is compact, there exist $l_{2}>l_{1} \geq N_{0}$, such that

$$
d\left(f^{l_{2}}(x), f^{l_{1}}(x)\right)<\frac{\delta^{\prime}}{2},
$$

where $\delta^{\prime}=\delta\left(\frac{\varepsilon}{2}\right)$ is as in the shadowing theorem for hyperbolic set. So $\bar{\xi}=\left\{f^{l_{1}}(x), f^{l_{1}+1}(x), \cdots, f^{l_{2}-1}(x)\right\}$ is a periodic $\delta^{\prime}$-pseudo-orbit of $f$ in $U_{i}$. By shadowing theorem for hyperbolic sets, there is $z \in \Lambda_{i}$, which $\frac{\varepsilon}{2}$-shadows $\bar{\xi}$. This shows that

$$
o(z, f) \subset N_{\frac{\varepsilon}{2}}(o(x, f)) .
$$

But $\Lambda_{i}$ has the inverse shadowing property with respect to classes $\theta_{s}$ and $\theta_{c}$. Thus there exists $\delta>0$ such that for any continuous $\delta$-method $\varphi$, we can find a pseudo-orbit $\xi=\left\{x_{k}\right\}_{k \in Z} \in G \Psi$ satisfying

$$
d\left(f^{k}(z), x_{k}\right)<\frac{\varepsilon}{2}, k \in Z .(* *)
$$

Inequalities $(*)$ and $(* *)$ show that $\xi \subset N_{\varepsilon}(o(x, f))$. This complete the proof of theorem 1 .

Theorem 2 Let $f$ be an $\Omega$-stable diffeomorphism and $\Omega(f)=\Lambda_{1} \cup \Lambda_{2} \cup \cdots \cup \Lambda_{k}$ be the Smale Spectral Decomposition such that $\left\{\Lambda_{i}, i=1, \cdots, k\right\}$ be fix point sources or sinks. Then $f$ has the weak inverse shadowing property with respect to class $\mathcal{T}_{0}$ in $M-$ Fix $(f)$, where Fix $(f)$ is set of fix points of $f$.

Proof. Let $\varepsilon>0$ and $x \in M$ be arbitrary that is not fix point and $\left\{U_{i}, i=1, \cdots, k\right\}$ be open neighborhoods of $\left\{\Lambda_{i}, i=1, \cdots, k\right\}$ respectively with diameter less than $\varepsilon$. Lemma 1 shows that there exists $N_{0}^{\prime} \in N$ and $U_{i}$ and $U_{j}$ for some $1 \leq i, j \leq k$, such that

$$
\left\{f^{N_{0^{\prime}+k}}(x)\right\}_{k \geq 0} \subset U_{i},
$$

and

$$
\left\{f^{-N_{0^{\prime}}+k}(x)\right\}_{k \geq 0} \subset U_{j},
$$

Note that $U_{i}$ is a neighborhood of fix point sink and $U_{j}$ is a neighborhood of fix point source. Choose 


$$
0<\delta_{0}<\frac{\min \left\{\operatorname{dim}\left(N_{\varepsilon^{\prime}}\left(\Lambda_{i}\right)-f\left(N_{\varepsilon^{\prime}}\left(\Lambda_{i}\right)\right)\right) \text { and } \operatorname{dim}\left(N_{\varepsilon^{\prime}}\left(\Lambda_{j}\right)-f^{-1}\left(N_{\varepsilon^{\prime}}\left(\Lambda_{j}\right)\right)\right)\right\}}{2}
$$

such that

$$
d(f(x), f(z))<\frac{\varepsilon^{\prime}}{4}
$$

for every $x, z \in M$ with $d(x, z)<\delta$, where

$$
\begin{aligned}
& 0<\varepsilon^{\prime}<\min \left\{\left\{\operatorname{diam}\left(U_{i}\right), i=1, \cdots, k\right\}, \frac{\varepsilon}{2}\right\} \\
& \text { and } f\left(N_{\varepsilon^{\prime}}\left(\Lambda_{i}\right)\right) \subset\left(N_{\varepsilon^{\prime}}\left(\Lambda_{i}\right)\right) \\
& \text { and } f^{-1}\left(N_{\varepsilon^{\prime}}\left(\Lambda_{j}\right)\right) \subset N_{\varepsilon^{\prime}}\left(\Lambda_{j}\right) .
\end{aligned}
$$

This shows that if $\xi=\left\{x_{k}\right\} \subset M$ is a $\delta_{0}$-pseudo orbit and $x_{l} \in N_{\varepsilon^{\prime}}\left(\Lambda_{i}\right)\left(x_{l} \in N_{\varepsilon^{\prime}}\left(\Lambda_{j}\right)\right)$

then $\left\{x_{n}\right\}_{n \geqslant l} \subset N_{\varepsilon^{\prime}}\left(\Lambda_{i}\right)\left(\left\{x_{n}\right\}_{n \leqslant l} \subset N_{\varepsilon^{\prime}}\left(\Lambda_{j}\right)\right)$. there exists $N_{0} \in N$ such that

$$
\left\{f^{N_{0}+k}(x)\right\}_{k \geq 0} \subset N_{\varepsilon^{\prime}}\left(\Lambda_{i}\right)
$$

and

$$
\left\{f^{-N_{0}-k}(x)\right\}_{k \geq 0} \subset N_{\varepsilon^{\prime}}\left(\Lambda_{j}\right) .
$$

Choose $\delta_{N_{0}+1}<\delta_{N_{0}}<\cdots<\delta_{1}<\delta_{0}$ such that if $d(x, y)<\delta_{i}$ for $i=N_{0}+1, N_{0}, \cdots, 1$ then

$$
\begin{aligned}
& d(f(x), f(y))<\frac{\delta_{i-1}}{N_{0}+1} \\
& \text { and } d\left(f^{-1}(x), f^{-1}(y)\right)<\frac{\delta_{i-1}}{N_{0}+1}
\end{aligned}
$$

And also $\frac{\delta_{i}}{N_{0}+1}+\delta_{N_{0}+1}<\delta_{i}$ for $i=N_{0}+1, N_{0}, \cdots, 1$.

So for any $\delta_{N_{0}+1}$-pseudo orbit

$$
\left\{x_{-N_{0}-1}, x_{-N_{0}}, \cdots, x, x_{1}, \cdots, x_{N_{0}+1}\right\}
$$

we have

$$
d\left(f^{i}(x), x_{i}\right)<\frac{\varepsilon^{\prime}}{2}, \quad i=-N_{0}-1, \cdots, N_{0}+1 .
$$

Now for any $\left(\delta_{N_{0}+1}\right)$-method $\varphi$, by regarding the process of choosing $\delta_{N_{0}+1}$ and (4), (5), (6) we have $\varphi(x) \subset N_{\varepsilon}(o(x, f))$, and this completes the proof of theorem 2 .

The following example shows that an $\Omega$-stable maybe has not the weak inverse shadowing property with respect to class $\mathcal{T}_{0}$ in its fix point.

Example. Represent $\mathbb{T}^{2}$ as the sqare $[-2,2] \times[-2,2]$, with identified opposite sides. Let $g: \mathbb{T}^{2} \rightarrow \mathbb{T}^{2}$ be a diffeomorphism with the following properties:

The nonwandering set $\Omega(g)$ of $g$ is the union of 4 hyperbolic fixed points, that is, $\Omega(g)=\left\{p_{1}, p_{2}, p_{3}, p_{4}\right\}$, where $p_{1}$ is a source, $p_{2}$ is a sink, and $p_{3}, p_{4}$ are saddles;

$$
\begin{aligned}
& W^{u}\left\{p_{4}\right\} \cup\left\{p_{3}\right\}=W^{s}\left(p_{3}\right) \cup\left\{p_{4}\right\} \\
& =[-2,2] \times\{0\}, W^{s}\left(p_{4}\right) \\
& =\{1\} \times(-2,2), W^{u}\left(p_{3}\right)=\{-1\} \times(-2,2),
\end{aligned}
$$

where $W^{s}\left(p_{i}\right)$ and $W^{u}\left(p_{i}\right)$ are the stable and unstable manifolds, respectively.

There exist neighborhoods $U_{3}, U_{4}$ of $p_{3}, p_{4}$ such that $g(x)=p_{i}+D_{p_{i}} g\left(x-p_{i}\right)$ for $x \in U_{i}$.

The eigenvalues of $D_{p_{3}} g$ are $-\mu, v$ with $0<v<1<\mu$, and the eigenvalues of $D_{p_{4}} g$ are $-\lambda, \kappa$ with $0<\lambda<1<\kappa$.

Plamenevskaya [16] showed that $g$ has the weak shadowing property if and only if the number $\frac{\log \lambda}{\log \mu}$ is irrational. Note that $g$ does not have the shadowing property. We can see that $g$ does not have the weak inverse shadowing property with respect to class $\mathcal{T}_{0}$ as well (Note that the number $\frac{\log \lambda}{\log \mu}$ is not necessary irrational). For any $0<\varepsilon<\frac{1}{4}$, let $0<\delta<\varepsilon$ be the number of the weak inverse shadowing property of $g$. Construct a $\delta$-method as following:

$$
\begin{aligned}
& \varphi\left(p_{4}\right) \\
& =\left\{\cdots, f^{-2}\left(p_{4}\right), f^{-1}\left(p_{4}\right), p_{4}, x_{0}, f\left(x_{0}\right), f^{2}\left(x_{0}\right), \cdots\right\},
\end{aligned}
$$

where $x_{0} \in(-1,1) \times\{0\} \subset W^{u}\left(p_{4}\right)$ and $d\left(p_{4}, x_{0}\right)<\delta$. For every $x \neq p_{4}$, define

$$
\varphi(x)=\left\{\cdots, f^{-2}(x), f^{-1}(x), x, f(x), f^{2}(x), \cdots\right\},
$$

\section{Relation between Minimality and Weak Inverse Shadowing Property with Respect to Class $\mathcal{T}_{0}$}

A homeomorphism $f: X \rightarrow X$ is called minimal if $f(A)=A, A$ closed, implies either $A=M$ or $A=\phi$. It is easy to see that $f$ is minimal if and only if $\overline{o(x, f)}=X$ for every $x \in X$.

A homeomorphiosm $f$ is said to be chain transitive if for every $x, y \in X$ and $\delta>0$ there are $\delta$-pseudoorbits from $x$ to $y$ and from $y$ to $x$.

The following example shows that there exists homeomorphiosms $f$ with inverse shadowing property with respect to class $\mathcal{T}_{0}$ which is not minimal. 
Example. Let $X:=\left\{x=\left\{x_{n}\right\}_{-\infty}^{\infty}: x_{n} \in\{0,1\}\right\}$ with metric

$$
d(x, y)=\left\{\begin{array}{cc}
2 & \text { if } x_{0} \neq y_{0} \\
\frac{1}{\min \left\{|k|: x_{k} \not y_{k}\right\}} & \text { if } x_{0}=y_{0}
\end{array}\right.
$$

Let $\pi:\{-n,-n+1, \cdots, n\} \rightarrow\{-n,-n+1, \cdots, n\}$ be a permutation of the set $\{-n,-n+1, \cdots, n\}$ for some $n \in \mathbb{N}$. Let $f_{n}(x)=x_{\pi(i)}$ if $-n \leq i \leq n$, and $x_{i}$ otherwise.

$f_{n}$ is a homeomorphism and every point of $X$ is a periodic point for $f_{n}$. We claim $f_{n}$ has weak inverse shadowing property with respect to class $\mathcal{T}_{0}$.

Proof of claim. Given $\varepsilon>0$ choose $N_{0}>n$ such that $\frac{1}{N_{0}}<\frac{\varepsilon}{2} . d(x, y)<\frac{1}{N_{0}}$ if and only if $x_{i}=y_{i}$ where $-N_{0} \leq i \leq N_{0}$. Let $\delta=\frac{1}{N_{0}}$ and $\varphi$ be a $\delta$-method. Let $x, y, z \in X$, then $d\left(f_{n}(x), y\right)<\delta$ implies $f_{n}(x)_{i}=y_{i}$ for $-N_{0} \leq i \leq N_{0}$ and hence by definition of $f_{n}, f_{n}^{2}(x)_{i}=f_{n}(y)_{i}$ for $-N_{0} \leq i \leq N_{0}$. Also $d\left(f_{n}(y), z\right)<\delta$ implies $f_{n}(y)_{i}=z_{i}$ for $-N_{0} \leq i \leq N_{0}$. Hence if $d\left(f_{n}(x), y\right)<\delta$ and $d\left(f_{n}(y), z\right)<\delta$ then $f_{n}^{2}(x)_{i}=z_{i}$ for $-N_{0} \leq i \leq N_{0}$, and so $d\left(f_{n}^{2}(x), z\right)<\delta$. Using this procedure we will get $d\left(f_{n}^{i}(x), \varphi(x)_{i}\right)<\delta$ for $i \geq 0$. A similar reasoning with having in mind that $f_{n}$ is a homeomorphism proves that $d\left(f_{n}^{i}(x), \varphi(x)_{i}\right)<\delta$ for $i \leq 0$. Hence $d\left(f_{n}^{i}(x), \varphi(x)_{i}\right)<\delta$ for $i \in \mathbb{Z}$ and $f_{n}$ has inverse shadowing property with respect to $\mathcal{T}_{0}$. It is easy to see that $f_{n}$ is not minimal.

Theorem 3 Let $f$ be a chain transitive homeomorphism on compact metric space $X$. Then $f$ is minimal if and only if $f$ has weak inverse shadowing property with respect to class $\mathcal{T}_{0}$.

Proof. Suppose that $f$ has weak inverse shadowing property with respect to class $\mathcal{T}_{0}$ and $z \in X$. Let $U$ be an open set in $X$. Choose $x_{0} \in U$ and $\varepsilon>0$ such that $N_{\varepsilon}\left(x_{0}\right) \subset U$. There is $\delta>0$ such that for each $\delta$-method $\varphi$, there is $y \in X$ such that

$$
\varphi(y) \subset N_{\frac{\varepsilon}{2}}(o(x, f))
$$

For every $x \in X$, there is a $\delta$-chain, $\left(x, x, \cdots, x_{l_{n}}, x_{0}\right)$ from $x$ to $x_{0}$. Consider

$$
\begin{aligned}
& \delta_{x}= \\
& \left\{\cdots, f^{-2}(x), f^{-1}(x), x, x_{1}, x_{2}, \cdots, x_{l_{n}}, x_{0}, f\left(x_{0}\right), \cdots\right\}
\end{aligned}
$$

as a $\delta$-pseudo-orbit, such that it's 0 -component be $X$. Construct a $\delta$-method $\varphi_{x_{0}}$ such that $\varphi_{x_{0}}(x)=\delta_{x}$. Hence there is $y \in X$ such that $\varphi_{x_{0}}(y) \subset N_{\frac{\varepsilon}{2}}(o(z, f))$, and so $d\left(x_{0}, f^{l}(z)\right)<\frac{\varepsilon}{2}$ for some $l \in \mathbb{Z}$. Therefore $o(z, f) \cap U \neq \varnothing$. This shows that each orbit of $X$ is dense in $X$ and so $f$ is minimal. The converse i.e. to see that each minimal homeomorphism has weak inverse shadowing property with respect to class $\mathcal{T}_{0}$, is obvious.

\section{Relation between Expansivity and Inverse Shadowing Property with Respect to Class $\mathcal{T}_{\mathbf{0}}$}

A homeomorphism $f$ on metric space $(X, d)$ is said expansive if there exists constant $e>0$ such that for every $x, y \in X,(x \neq y)$ there exists integer number $N_{0}$ such that $d\left(f^{N_{0}}(x), f^{N_{0}}(y)\right)>e$.

Theorem 4 If homeomorphism $f$ on metric space $(X, d)$ has the inverse shadowing property with respect to class $\mathcal{T}_{0}$, then $f$ is not expansive.

Proof. Suppose that $f$ is expansive and has the inverse shadowing property with respect to class $\mathcal{T}_{0}$. Let $e>0$ be as in definition of expansivity and $0<\delta<e$ be such that for any $\delta$-method $\varphi$ in $\mathcal{T}_{0}$ and any point $x \in X$ there exists a point $y \in X$ for which $d\left(f^{n}(x), \varphi(y)_{n}\right)<e, n \in \mathbb{Z}$.

Let $x_{0} \in X$ be arbitrary. Choose $y_{0} \neq x_{0}$ such that $d\left(x_{0}, y_{0}\right)<\delta$ and $d\left(f\left(x_{0}\right), f\left(y_{0}\right)\right)<\delta$. Construct a $\delta$-method $\varphi$ as following.

For any $x \neq x_{0}$ define

$$
\begin{aligned}
& \varphi(x) \\
& =\left\{\cdots, f^{-2}(x), f^{-1}(x), x, f(x), f^{2}(x), \cdots\right\}
\end{aligned}
$$

and

$$
\begin{aligned}
& \varphi\left(x_{0}\right) \\
& =\left\{\cdots, f^{-2}\left(y_{0}\right), f^{-1}\left(y_{0}\right), x, f\left(y_{0}\right), f^{2}\left(y_{0}\right), \cdots\right\}
\end{aligned}
$$

Since $f$ has the inverse shadowing property with respect to class $\mathcal{T}_{0}$, for $x_{0}$ there exists $y \in X$ such that

$$
d\left(f^{n}\left(x_{0}\right), \varphi(y)_{n}\right)<e, n \in \mathbb{Z} .
$$

By regarding to choose of $\delta$-method $\varphi$, we have

$$
d\left(f^{n}\left(x_{0}\right), f^{n}(y)\right)<e \text { for } n \in \mathbb{Z}
$$

for some $y \neq x_{0}$, that contradicts the expansivity of $f$. This completes the proof of theorem.

\section{Conclusion}

In this paper we showed that an $\Omega$-stable diffeomorphism $f$ has the weak inverse shadowing property with respect to classes of continuous method $\theta_{s}$ and $\theta_{c}$ 
and some of the $\Omega$-stable diffeomorphisms have weak inverse shadowing property with respect to classes $\mathcal{T}_{0}$. In addition we studied relation between minimality and weak inverse shadowing property with respect to class $\mathcal{T}_{0}$ and relation between expansivity and inverse shadowing property with respect to class $\mathcal{T}_{0}$.

\section{REFERENCES}

[1] R. Corless and S. Plyugin, "Approximate and Real Trajectories for Generic Dynamical Systems," Journal of Mathematical Analysis and Applications, Vol. 189, No. 2, 1995, pp. 409-423. doi:10.1006/jmaa.1995.1027

[2] P. Diamond, P. Kloeden, V. Korzyakin and A. Pokrovskii, "Computer Robustness of Semihypebolic Mappings," Random and computational Dynamics, Vol. 3, 1995, pp. 53-70.

[3] P. Kloeden, J. Ombach and A. Pokroskii, "Continuous and Inverse Shadowing," Functional Differential Equations, Vol. 6, 1999, pp. 137-153.

[4] K. Lee, "Continuous Inverse Shadowing and Hyperbolic," Bulletin of the Australian Mathematical Society, Vol. 67, No. 1, 2003, pp. 15-26. doi:10.1017/S0004972700033487

[5] S. Yu. Pilyugin, "Inverse Shadowing by Continuous Methods," Discrete and Continuous Dynamical Systems, Vol. 8, No. 1, 2002, pp. 29-28. doi:10.3934/dcds.2002.8.29

[6] P. Diamond, Y. Han and K. Lee, "Bishadowing and Hyperbolicity," International Journal of Bifuractions and Chaos, Vol. 12, 2002, pp. 1779-1788.
[7] T. Choi, S. Kim and K. Lee, "Weak Inverse Shadowing and Genericity," Bulletin of the Korean Mathematical Society, Vol. 43, No. 1, 2006, pp. 43-52.

[8] B. Honary and A. Zamani Bahabadi, "Weak Strictly Persistence Homeomorphisms and Weak Inverse Shadowing Property and Genericity," Kyungpook Mathematical Journal, Vol. 49, 2009, pp. 411-418.

[9] R. Gu, Y. Sheng and Z. Xia, "The Average-Shadowing Property and Transitivity for Continuous Flows," Chaos, Solitons, Fractals, Vol. 23, No. 3, 2005, pp. 989-995.

[10] L.-F. He and Z.-H. Wang, "Distal Flows with the Pseudo Orbit Tracing Property," Chinese Science Bulletin, Vol. 39, 1994, pp. 1936-1938.

[11] K. Kato, "Pseudo-Orbits and Stabilities Flows," Memoirs of the Faculty of Science Kochi University Series A Mathematics, Vol. 5, 1984, pp. 45-62.

[12] M. Komouro, "One-Parameter Flows with the PseudoOrbit Tracing Property," Mathematics and Statistics, Vol. 98, No. 3, 1984, pp. 219-253. doi:10.1007/BF01507750

[13] C. Robinson, "Dynamical Systems: Stability, Symbolic Dynamics, and Chaos," CRC Press, Boca Raton, 1998.

[14] S. Yu. Pilyugin, "Introduction to Structurally Stable Systems of Differential Equations," Birkhauser Verlag, Boston, 1992. doi:10.1007/978-3-0348-8643-7

[15] I. P. Malta, "Hyperbolic Birkhoff Centers," Transactions of the American Mathematical Society, Vol. 262, 1980, pp. 181-193. doi:10.1090/S0002-9947-1980-0583851-4

[16] O. B. Plaenevskaya, "Weak Shadowing for Two-Dimensional Diffeomorphism," Vestnik St. Petersburg University: Mathematics, Vol. 31, 1998, pp. 49-56. 